\title{
A 3-D indoor AOA localization system based on
}

\section{geographic information database}

\author{
Ze Zheng ${ }^{1, a^{*}}$, Zhanliang Zhang ${ }^{2, b}$ and Guoru Ding ${ }^{1, c}$ \\ ${ }^{1}$ College of Communications Engineering, PLA University of Science and Technology, China \\ ${ }^{2}$ College of Field Engineering, PLA University of Science and Technology, China \\ a403858708@qq.com, ${ }^{b} 370504970 @ q q . c o m$, dingguoru@gmail.com
}

Keywords: indoor localization; GIDB; 3-D

Abstract. Angle-of-arrival (AOA) method is one of the popular methods for indoor localization, which plays well in the direct path case. However, the indoor environment is more complex, which means the direct path does not exist in most cases. To tackle this issue, in this paper, we propose a 3-D indoor localization system based on geographic information database (GIDB) utilizing AOA to adapt to the complex environment. This system takes geographic information as prior information. Based on the information, this system knows which area measured source belongs to, which means whether there exists direct path or not is able to be detected, which help localize the position of measured source. Simulation shows that the localization error is close to that of AOA under the scenario direct path exists in theory.

\section{Introduction}

Indoor localization plays an increasingly important role nowadays. Angle-of-arrival (AOA) is a traditional indoor localization method, whose accuracy may be influenced by shadowing, multipath reflection and so on [1]-[4]. One main problem of AOA is that there must be direct path between measured source (MS) and antenna array, which is usually harsh in complex indoor environment.

In this paper, we propose an indoor localization system based on geographic information database (GIDB), which trying to resolve the problem mentioned above utilizing the prior information provided by GIDB. The contributions of this paper are as follows:

1) An indoor localization system architecture based on GIDB is proposed in this paper. The GIDB includes obstacle database, shadow database and area database.

2) Based on the prior information from GIDB, a 3-D localization approach utilizing AOA is proposed, whose accuracy is close to that of AOA under the scenario direct path exists in theory, no matter where there exist direct path or not.

The following contents of this paper are as follows. In system model, we discuss the research scenario and some assumption in this scenario. The database architecture and the localization problem in this system is discussed in system architecture. The relative method is proposed in localization and simulation. At last, some conclusions will be drawn.

\section{System model}

Scenario. Figure 1 shows the top view of our meeting room, which is the simulation scenario in this paper. We only focus on the influence of the table in the center of this meeting room instead of other objects. So the actual research scenario is changed from the left one to the right one in figure 1, where two antenna arrays are set on the left and right walls. 


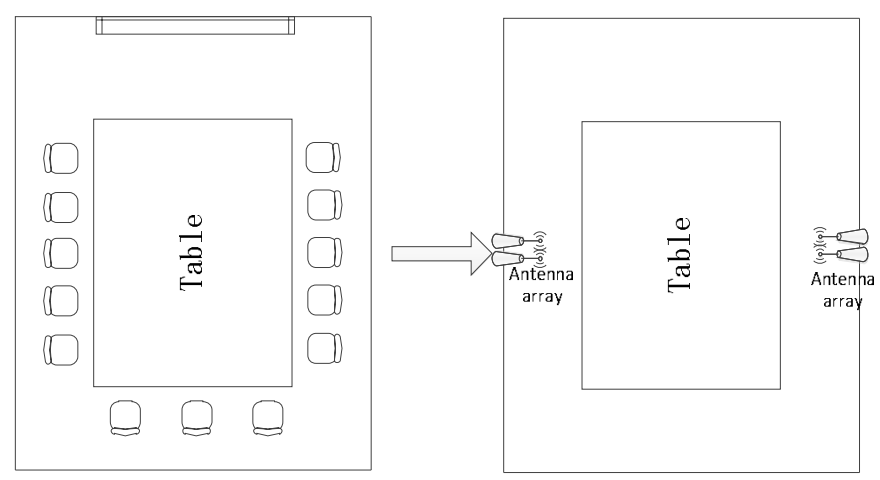

Fig.1. meeting room

We assume that the influence of this table is obstructing the signal path. Considering the signal attenuation, we also assume that antenna array is only able to receive the signal from direct path and one-reflection path. The signal of two-more-reflection path is so weak that we take it as noise.

Angle Measuring Assumption. Comparing to the signal strength in the direct path, that in one-reflection path is much smaller, which may attenuate about $10 \mathrm{~dB}$. When two signals arrive antenna array, if the differ between their signal strength is big, perhaps only the angle of the stronger signal arrival is able to be calculated. The reason is that the side lobe near the main lobe of the weak signal may cover its main lobe. We simulate that the two angles of signal arrival are -60 degrees and 60 degrees, and the signal strength of 60 degrees is $13 \mathrm{~dB}$ larger than that of -60 degrees. Figure 2 illustrates the lobe pattern and the relative result of MUSIC algorithm in the case mentioned above.

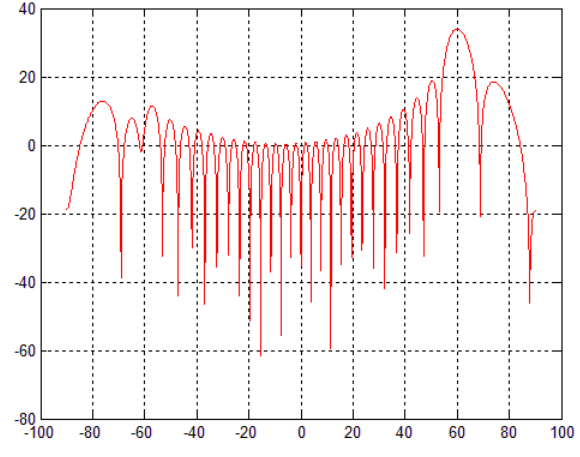

Fig.2-1. Lobe pattern

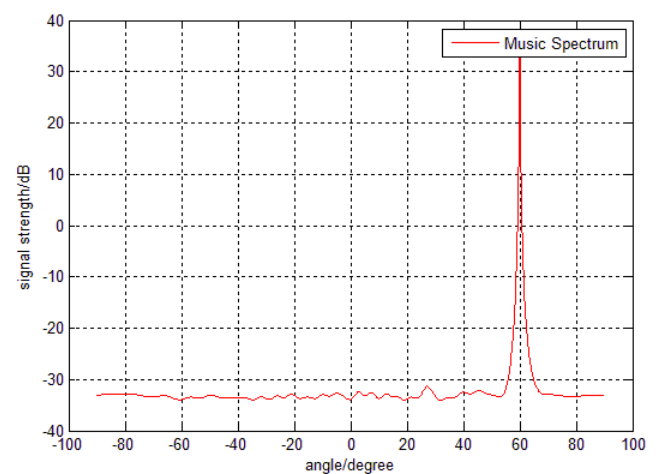

Fig.2-2. MUSIC algorithm

Based on this simulation result, three assumptions about angle measurement are as follows:

1) If there is a direct path between MS and antenna array, the antenna array is only able to calculate the direction of signal arrival in direct path. Because the signal strength of direct path is large enough to cover that of one-reflection path.

2) If there is a one-reflection path between MS and antenna array instead of direct path, the direction of signal arrival in one-reflection path is able to be calculated.

3) If there is no direct path or one-reflection path between MS and antenna array, due to the weakness of signal strength in two-more-reflection path, no direction of signal arrival can be calculated.

The Position and Capability of Antenna Array. In 3-D localization, one antenna array is able to return two angles, pitch angle and direction angle, such as longitude and latitude [5]. If these two 
antenna arrays are set in an appropriate position, the pitch angle and the direction angle are able to be defined respectively as follows.

1) In the side view of this meeting room (figure 3-1), we define the angle of signal path each antenna array received is pitch angle in this 2-D plane, which may determine the width and the height of each MS. $\beta_{1}$ and $\beta_{2}$ are defined as the received angle of the left antenna array and that of right one respectively.

2) Similarly, in the top view of this scenario, $\alpha_{1}$ and $\alpha_{2}$ are defined as the direction angles of each antenna array in this 2-D plane, which is able to determine the length and the width of each MS.

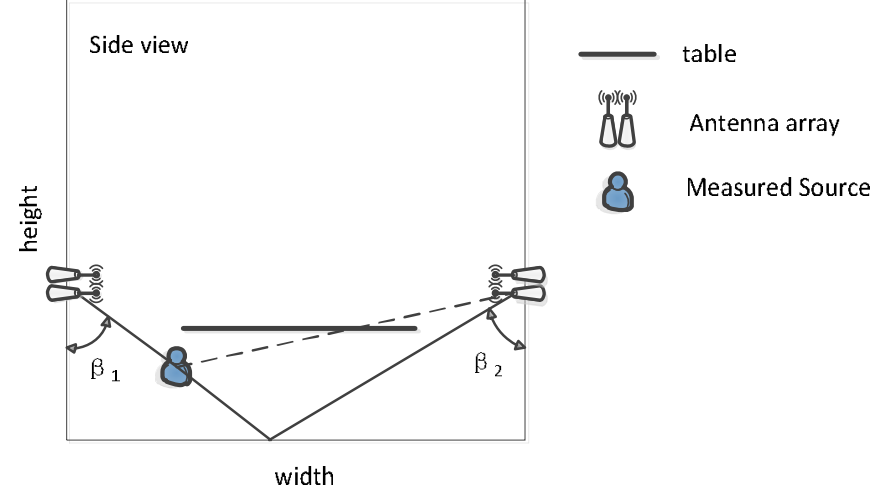

Figure.3-1. $\beta_{1}$ and $\beta_{2}$ are pitch angles

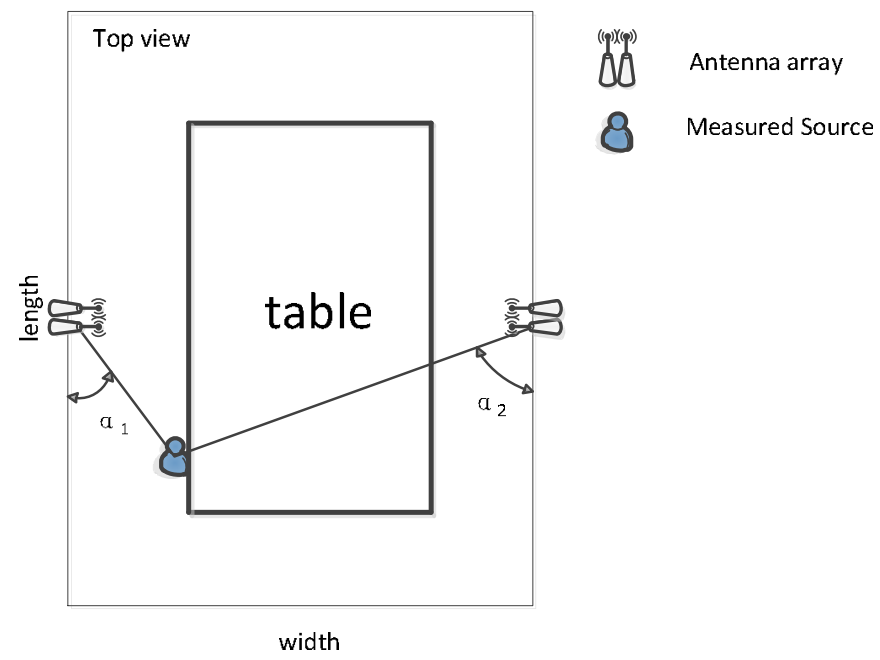

Figure.3-2. $\alpha_{1}$ and $\alpha_{2}$ are direction angle

Considering every position of this scenario is able to be measured, there must be direct path or one-reflection path between MS and these two antenna arrays. Thus, there must be direct path between antenna array and its opposite angle, or the signal in a part of area may not arrive at the antenna array based on the three assumptions mentioned above, which is illustrated in the gray area of figure 4.

Therefore, if we meet the requirement, the whole area of side view is divided into 5 parts, which is illustrated in figure 5. Each point of area 1 and area 2 owns two direct paths between the two arrays and itself. Similarly, those of area 3 and area 4 own one direct path and one one-reflection path. And those of area 5 own two one-reflection paths. 


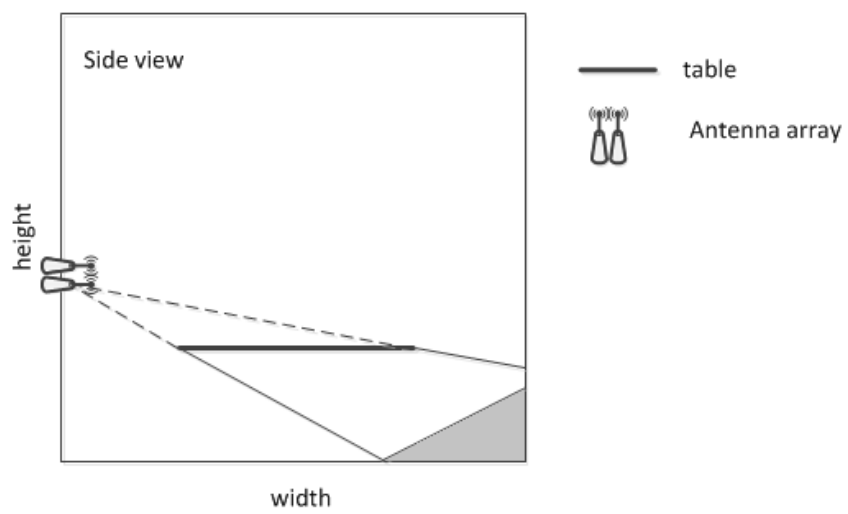

Fig.4. area of blind measuring

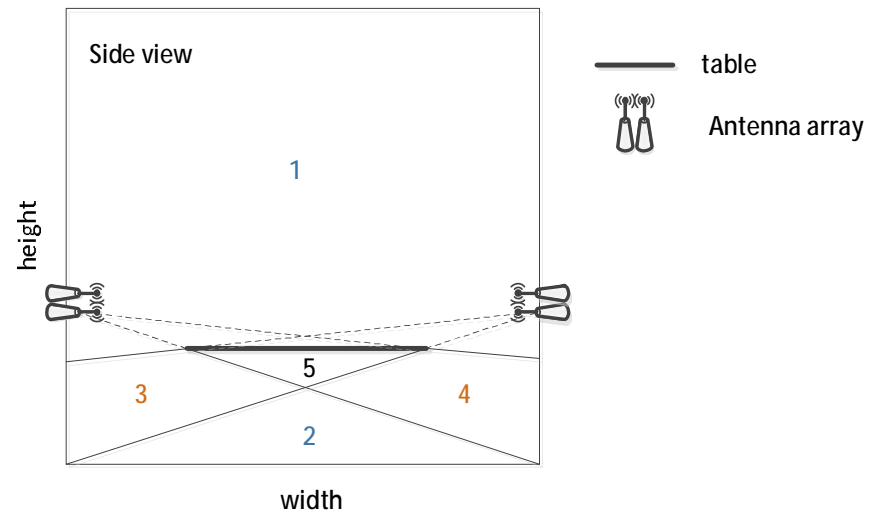

Fig.5. five areas of side view

\section{System architecture}

The system includes three parts, antenna array, data handle center (DHC) and GIDB illustrated in figure 6. DHC controls antenna arrays and queries GIDB to acquire prior information. Antenna array measure each direction of signal arrival. GIDB provides prior information according to the angles transmitted from DHC.

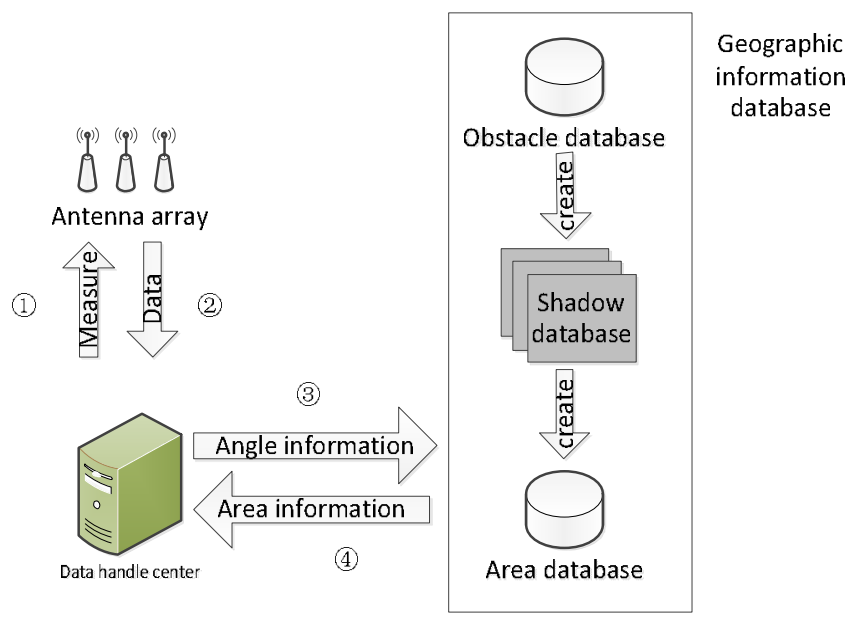

Fig.6. system architecture

The four measured steps are as follows.

1) DHC requires antenna array to measure MS.

2) Antenna array returns a group of measured data to DHC. DHC calculate the direction angle vector $\left[\alpha_{1}, \alpha_{2}\right]$ and the pitch angle vector $\left[\beta_{1}, \beta_{2}\right]$. 
3) DHC transmits the pitch angle vector $\left[\beta_{1}, \beta_{2}\right]$ to GIDB so as to query the relative prior information.

4) GIDB returns the prior information to DHC. Based on the information, DHC calculate the final position of MS.

Generally, GIDB includes two parts of information, spatial information and attribute information. Spatial information records the spatial position, which is a $3-\mathrm{D}$ vector $[x, y, z]$ in this system. Attribute information refers to the attribute of the relative spatial position, which is determined by designer. According to the attribute information, GIDB includes three sub databases in this system, obstacle database (ODB), shadow database (SDB) and area database (ADB).

1) ODB: ODB records whether it is obstacle or not in each position. In this scenario, the positions of table are obstacles, which is recorded ' 1 ' in ODB. And others are recorded ' 0 ' in ODB.

2) SDB: SDB records whether there is direct path between each position and relative antenna array based on ODB. Thus, the number of SDB is determined by the number of antenna array, which is two in this system. If there is direct path, we record ' 1 ' in the relative SDB. On the contrary, ' $O$ ' is recorded. Therefore, every position of area 4 and area 5 in figure 5 is recorded ' $O$ ' in the SDB of left antenna array. Similarly, those of area 3 and area 5 are recorded ' $O$ ' in the SDB of right antenna array.

3) $\mathrm{ADB}$ : In $\mathrm{ADB}$, we record which area each position belongs to according to all SDBs. In this system, five areas are divided in figure 5. The prior information DHC needs is provided by this sub database.

\section{Localization and simulation}

Our goal is to detect the position vector $[x, y, z]$ in 3-D localization. We assume that $x$ represents the value of length in this scenario, y represents that of width, and $\mathrm{z}$ is the value of height. In the top view, we are able to detect $[x, y]$ by the direction angle vector $\left[\alpha_{1}, \alpha_{2}\right]$, and $[y, z]$ can be detected by the pitch angle vector $\left[\beta_{1}, \beta_{2}\right]$ in the side view in theory.

Localization of Direction Angle. In the plane of top view in figure 3-2, the position of each antenna array is known, which we assume $\left[\mathrm{x}_{\mathrm{a} 1}, \mathrm{y}_{\mathrm{a} 1}\right]$ and $\left[\mathrm{x}_{\mathrm{a} 2}, \mathrm{y}_{\mathrm{a} 2}\right]$ respectively. The direction angle $\alpha_{i}$ can be transformed into the relative gradient $\gamma_{i}$. At last, we solve the point slope equations of the two signal path to obtain the $\left[x_{M S}, y_{M S}\right]$ of MS.

Localization of Pitch Angle.Firstly, in the side view, we research the distribution of the five areas in angle space. Thus, we evenly get some points in the plane of side view. The distribution of each area in angle space is illustrated in figure 7. 


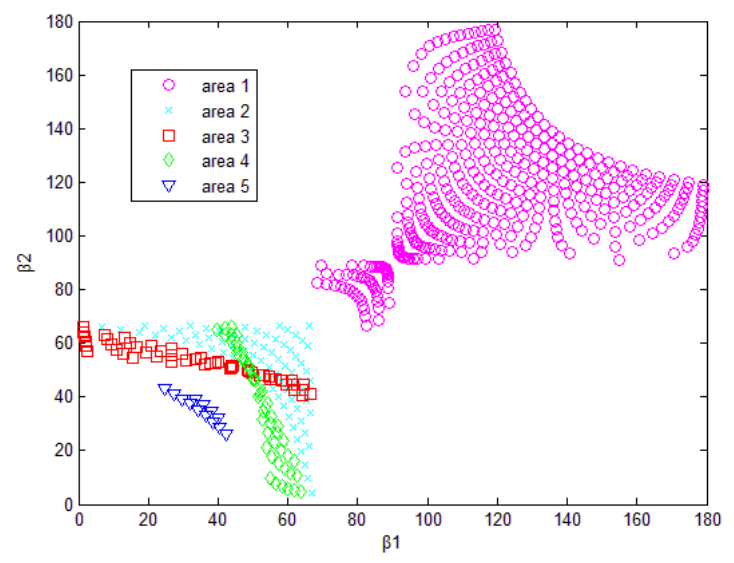

Fig.7. Distribution of each area in angle space

According to figure 7, all areas in angle space are continuous in the side view. Therefore, if the $\mathrm{ADB}$ records the contour of each area in angle space as the prior information, when DHC calculate $\left[\beta_{1}, \beta_{2}\right]$ of MS, which area of this vector belongs to is known based on the prior information provided by ADB. However, only area 1 and area 5 do not overlap with other areas. The other three areas have a part of region that overlaps each other, which means the system could not distinguish which area $\left[\beta_{1}, \beta_{2}\right]$ belongs to when it fall in the overlap area.

In order to solve this problem, the information of $\left[x_{M S}, y_{M S}\right]$ calculated according to direction angle is utilized. If which area $\left[\beta_{1}, \beta_{2}\right]$ belongs to is known, the relative spatial coordinate $\left[y_{M S}, z_{M S}\right]$ is able to be calculated. The $y_{M S}$ is known in the step mentioned above, which means which area $\left[\beta_{1}, \beta_{2}\right]$ belongs to can be detected by $y_{M S}$. So the following theorem should be proved.

In angle space, the two points in the same position of the overlap area own different width, i.e., $\mathrm{y}_{\mathrm{ms}}$ in spatial space.

In order to proving the above theorem, its contrapositive is just have to be proved, i.e.,

In spatial space, the two points owning the same width are in different places in angle space.

This proposition can be divided into the following three steps to prove.

1) Obviously, the width of each point in area 3 is different with that in area 4.

2) For any two different points owning the equal width in area 2 and area 3, the angles of their direct paths are different, i.e., $\beta_{M S 1}$ and $\beta_{M S 2}$ in figure 8 , which means these two points are in different places in angle space.

3) The arbitrary two different points owning equal width in area 2 and area 4 are also different in angle space, which is similar to step 2.

To sum up, the localization in this system is divided into three steps.

1) $\left[x_{M S}, y_{M S}\right]$ of MS is calculated according to the direction angle $\left[\alpha_{1}, \alpha_{2}\right]$.

2) Based on the prior information, all possible $\left[y_{M S i}, z_{M S i}\right]$ are calculated, where $i$ represent the possible area.

3) Selecting the $\left[y_{M S j}, z_{M S j}\right]$ who is minimum differ between $y_{M S}$ and $y_{M S j}$, i.e.

$$
\left[y_{M s_{j}}, z_{M S_{j}}\right]=\operatorname{argmin}\left(\left(y_{M S}-y_{M S_{j}}\right)^{2}\right) \text { for all } \mathrm{j}
$$

The final localization coordinate is $\left[x_{M S}, y_{M S}, z_{M S j}\right]$. 


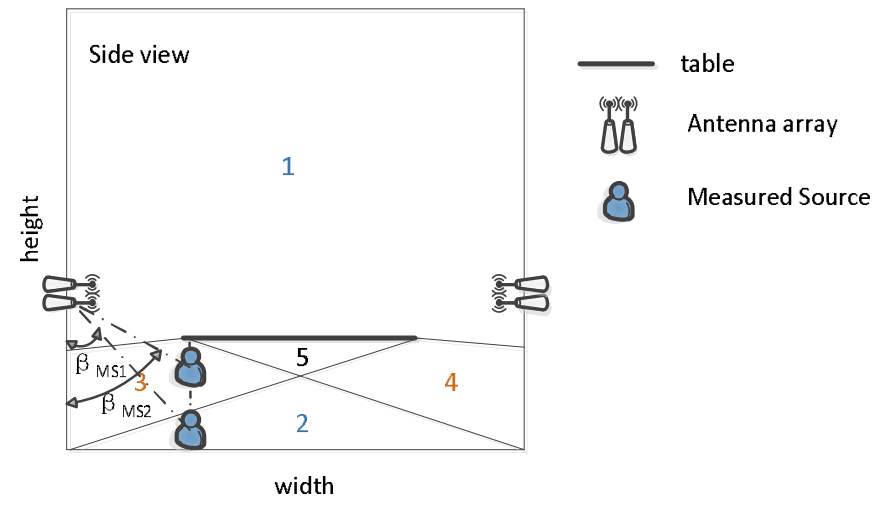

Fig. 8. $\beta_{M S 1}$ and $\beta_{M S 2}$ are different

In theory, the localization in 3-D scenario need three antenna arrays. However, we only utilize two arrays in this paper, which means the position in the line between these two arrays is not able to be localized.

Simulation. The size of this scenario is $150 * 50 * 50$ in the simulation. We simulate the localization method based on GIDB proposed in this paper and traditional AOA without GIDB respectively, which is shown in figure 9, where the horizontal coordinate is the area in figure 5, and the longitudinal coordinate is the error of localization in each area, which 1 represent the unit length of this scenario.

Due to no prior information in traditional AOA method, when facing the case of occlusion, the error is very large, such as in area 3, 4 and 5. However, when utilizing the prior information provided by GIDB, the error becomes very small, most of which is system error. The average error of the method based on GIDB is about 0.453 in the simulation.

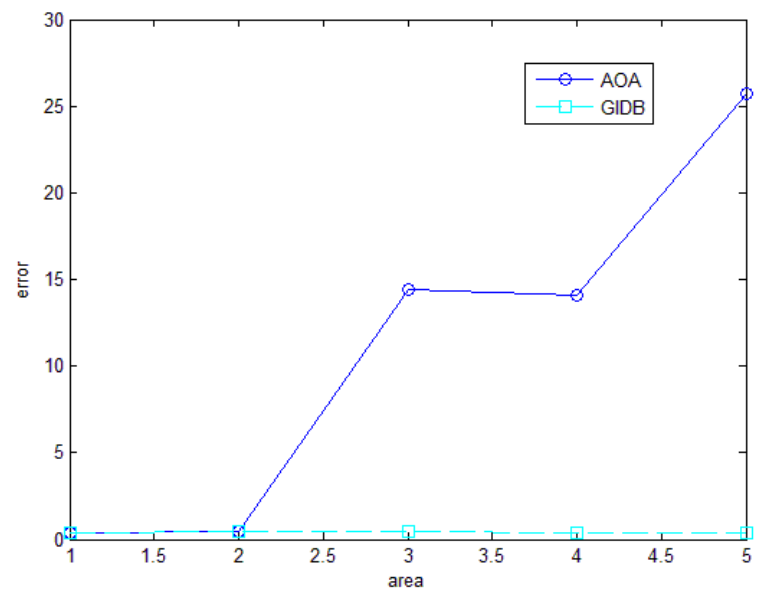

Fig.9. simulation of AOA and the method base on GIDB

\section{Conclusion}

In this paper, a 3-D localization system model utilizing geographic information database is proposed. And we apply it to a specific scenario in simulation. However, the concrete effect of this system is waiting to be measured in a real scenario. And how to use this system model in a more complex scenario is also a problem waited to be solved. Certainly, constructing a testbed of this system is also worth doing. 


\section{References}

[1] Liu H, Darabi H, Banerjee P, et al. Survey of wireless indoor positioning techniques and systems[J]. Systems, Man, and Cybernetics, Part C: Applications and Reviews, IEEE Transactions on, 2007, 37(6): 1067-1080.

[2] B. D. Van Veen and K. M. Buckley, "Beamforming: A versatile approach to spatial filtering," IEEE ASSP Mag., vol. 5, no. 2, pp. 4-24, Apr. 1988.

[3] P. Stoica and R. L. Moses, Introduction to Spectral Analysis. Englewood Cliffs, NJ: Prentice-Hall, 1997.

[4] B. Ottersten, M. Viberg, P. Stoica, and A. Nehorai, "Exact and large sample ML techniques for parameter estimation and detection in array processing," in Radar Array Processing, S. S. Haykin, J. Litva, and T. J. Shepherd, Eds. New York: Springer-Verlag, 1993, pp. 99-151.

[5] Y. M. Chen, J. H. Lee, and C. C. Yeh, "Two-dimensional angle-of-arrival estimation for uniform planar arrays with sensor position errors," IEE Proc. F, Radar Signal Process., vol. 140, no. 1, pp. 37-42, Feb. 1993. 